\title{
Non-Fickian diffusion in colloidal glasses
}

\author{
M. H. J. Hagen and D. Frenkel \\ FOM Institute for Atomic and Molecular Physics, Kruislaan 407, 1098 SJ Amsterdam, The Netherlands \\ C. P. Lowe \\ Computational Physics, Delft University of Technology, Lorentzweg 1, 2628 CJ Delft, The Netherlands
}

(Received 10 November 1997; accepted 19 March 1998)

\begin{abstract}
We have studied numerically the decay of the self-dynamic structure factor (SDSF) for a small particle diffusing in a colloidal glass. We show that, in line with theoretical predictions, the super-Burnett coefficient (characterizing the deviation of the fourth moment of the single particle distribution from its Gaussian value) is finite. However, our results also show that large scale deviations from Fick's law of diffusion should still be easy to detect experimentally. These deviations take the form of the SDSF decaying algebraically rather than exponentially as Fick's law would predict. (C) 1998 American Institute of Physics. [S0021-9606(98)50924-5]
\end{abstract}

\section{INTRODUCTION}

Most theoretical treatments for problems involving mass transport are based on the classical diffusion equation

$$
\frac{\partial}{\partial t} p(\mathbf{r}, t)=D \nabla^{2} p(\mathbf{r}, t) .
$$

It describes the temporal evolution of the probability density at a position $\mathbf{r}, p(\mathbf{r}, t)$, in terms of a single coefficient, the diffusion constant $D$. Two steps are required to arrive at this result. First there is an exact relation between the microscopic current density $\mathbf{j}(\mathbf{r}, t)$ and the probability density

$$
\frac{\partial}{\partial t} p(\mathbf{r}, t)=-\boldsymbol{\nabla} \cdot \mathbf{j}(\mathbf{r}, t) .
$$

This is the continuity equation and follows directly from the microscopic equations of motion. Second, there is the constitutive relation between $\mathbf{j}(\mathbf{r}, t)$ and $p(\mathbf{r}, t)$ which, according to Fick's law, is

$$
\mathbf{j}(\mathbf{r}, t)=-D \boldsymbol{\nabla} p(\mathbf{r}, t) .
$$

Whereas the continuity equation, Eq. (2), is exact, the constitutive equation, Eq. (3), is phenomenological. In cases where the diffusion equation applies, the diffusion coefficient itself can be related to the mean squared displacement of a single "tagged" particle $\Delta(t)$ as follows

$$
D=\lim _{t \rightarrow \infty} \frac{\Delta(t)}{2 d t},
$$

where $d$ is the dimensionality. This is the Einstein definition of the diffusion coefficient. The mean squared displacement of a tagged particle can also be related to a correlation function of the microscopic particle velocity, $\mathbf{v}(t)$, namely the velocity autocorrelation function, $C_{v}(t)$

$$
\frac{\Delta(t)}{2 d t}=\int_{0}^{t} C_{v}\left(t^{\prime}\right) d t^{\prime}-\frac{1}{t} \int_{0}^{t} t^{\prime} C_{v}\left(t^{\prime}\right) d t^{\prime} .
$$

The velocity autocorrelation function $(\mathrm{VCF})$ is defined as

$$
C_{v}(t)=\frac{1}{d}\langle\mathbf{v}(0) \cdot \mathbf{v}(t)\rangle
$$

and, comparing Eqs. (4) and (5), can be directly related to the diffusion coefficient

$$
D=\lim _{t \rightarrow \infty} \frac{\Delta(t)}{2 d t}=\int_{0}^{\infty} C_{v}\left(t^{\prime}\right) d t^{\prime} .
$$

This gives a definition of the diffusion coefficient of a tagged particle in terms of its microscopic dynamics.

When the VCF in a hard sphere fluid was studied by computer simulation, ${ }^{1}$ it was found to decay with an algebraic "long-time tail" of the form $C_{v}(t) \sim 1 / t^{3 / 2}$. This contradicted theories current at the time and new mode-coupling and kinetic theories were required to explain the origin of the long-time tail. ${ }^{2,3}$ These theories showed, furthermore, that Fick's law was valid in a more limited sense than had been thought. One can consider the constitutive relation between flux and gradient [Eq. (3)] as being the first term in an expansion involving higher derivatives of the gradient. These higher order terms represent corrections to Fick's law. However, the same mode-coupling theories which explain the existence of long-time tails also predict that at some point in the expansion the coefficients which precede the higher order terms (the Burnett coefficients) diverge. Similar behavior was predicted for a system even simpler than a hard sphere fluid, namely the Lorentz gas. ${ }^{4}$ Lorentz gases are models in which a point particle moves through a fixed array of objects. As such they can be considered as generic models for diffusion in random static media. For Lorentz gases the expectation was for the VCF to decay as $C_{v}(t) \sim-1 / t^{d / 2+1}$, which has been confirmed by computer simulation. ${ }^{5}$

If one is addressing higher order corrections to Fick's law it is convenient to consider the single particle probability density, $P(\mathbf{r}, t)$. The function $P(\mathbf{r}, t)$ is defined as the probability that a particle, initially located at the origin, is at position $\mathbf{r}$ at some later time $t$. Furthermore, rather than studying $P(\mathbf{r}, t)$ itself, it is advantageous to consider its Fourier transform $F(\mathbf{k}, t)$, known as the self-dynamic structure 
factor (SDSF). The reasons for this are twofold. First it can be measured experimentally by several techniques. Second it is possible to express the SDSF in terms of the moments of $P(\mathbf{r}, t)$. This is most conveniently done by means of a cumulant expansion about a Gaussian. In terms of Cartesian components $k$ and $r$, the first two terms in the expansion are

$$
\begin{aligned}
F(k, t)= & \exp \left(-\frac{k^{2}}{2 !}\left\langle r(t)^{2}\right\rangle+\frac{k^{4}}{4 !}\left(\left\langle r(t)^{4}\right\rangle\right.\right. \\
& \left.\left.-3\left\langle r(t)^{2}\right\rangle^{2}\right)+O\left(k^{6}\right)\right) .
\end{aligned}
$$

The solution to the diffusion equation [Eq. (1)], for a particle starting from the origin at $t=0$ is a Gaussian centered on the origin so, for a Fickian process, only the first term is nonzero and thus $F(k, t)=\exp \left(-k^{2} D t\right)$. Higher order terms in Eq. (8) reflect deviations of $P(\mathbf{r}, t)$ from a Gaussian or, equivalently, deviations from Fick's law. A general form which accounts for the deviations of the first and second term in the cumulant expansion from Fickian behavior is

$$
\begin{aligned}
F(k, t)= & \exp \left(-k^{2} \int_{0}^{t} D\left(t^{\prime}\right) d t^{\prime}+k^{4}\right. \\
& \left.\times \int_{0}^{t} B\left(t^{\prime}\right) d t^{\prime}+O\left(k^{6}\right)\right),
\end{aligned}
$$

from which it is convenient to define a "time and wave vector dependent' diffusion coefficient, $D(k, t)$,

$$
D(k, t)=-\frac{d}{d t}\left(\frac{\ln F(k, t)}{k^{2}}\right)=D(t)-B(t) k^{2}+O\left(k^{4}\right) .
$$

If a process is Fickian then $D(k, t)$ reduces to the diffusion coefficient. By definition the functions $D(t)$ and $B(t)$, the time dependent diffusion and super-Burnett coefficients, respectively, are given by

$$
\begin{aligned}
& D(t)=\frac{1}{2 !} \frac{d}{d t}\left\langle r(t)^{2}\right\rangle \\
& B(t)=\frac{1}{4 !} \frac{d}{d t}\left(\left\langle r(t)^{4}\right\rangle-3\left\langle r(t)^{2}\right\rangle^{2}\right) .
\end{aligned}
$$

Mode-coupling theory predicts that for a three dimensional Lorentz gas both converge. Only when we consider the next $\left(k^{4}\right)$ term in Eq. (10) should we find a divergence. ${ }^{6}$ One of our aims here is to examine the super-Burnett coefficient in this type of system. According to Eq. (9) deviations from Fick's law should be negligible if $k^{2} \ll D(t) / B(t)$. So if $B(t)$ and $D(t)$ both approach constant values, deviations from Fick's law should be limited to wave vectors larger than some fixed value, or, equivalently, to a short period of time. Here we argue that this is not necessarily true.

If we assume that the diffusion and super-Burnett coefficients do converge as a function of time, then we expect to find an exponentially decaying self-dynamic structure factor. However, the fact that the higher Burnett coefficients diverge means that at any given value of $k$ the decay at long times is more complex. In fact, a numerical study of the self-dynamic structure factor for a Lorentz gas (in which the fixed objects excluded volume), showed that it too decayed algebraically. ${ }^{7}$ In three-dimensions the decay had the form $F(k, t) \sim 1 / t^{3 / 2}$. Although this seemed surprising, the mode-coupling theory contained in Ref. 6 actually predicted such an effect, although this was not appreciated at the time. Obviously, such a decay is not consistent with Eq. (8) meaning that the cumulant expansion for $F(k, t)$ in this type of system does not exist. This in turn implies that there are additional, nonanalytic terms in the expansion of the flux in terms of concentration gradients.

For the simulations reported in Ref. 7, systems containing a low volume fraction of fixed obstacles (10\%) were studied and the amplitude of the long-time tail was found to be independent of the magnitude of the wave vector $k$. This is in agreement with a theoretical prediction based on solving the random walk problem in the presence of a single fixed object. $^{8}$ Based on these simulations one would have concluded that the algebraic decay of the SDSF was unlikely to be observed experimentally. The reasons for this are twofold. First the magnitude of the long-time tail was very small, it could only be observed with the aid of a very accurate computational technique. Second, because the amplitude of the tail was independent of $k$, the effect became proportionately smaller for smaller wave vectors or, equivalently, at longer times. To see why, one must consider which wave vectors are relevant as time increases. By "relevant" we mean wave vectors which could in practice be observed decaying. If we examine Eq. (9) it is clear that, for a given wave vector, $F(k, t)$ decays over times of the order $1 /\left(k^{2} D\right)$. At shorter times the SDSF will hardly have decayed, at longer times it will be very small. Consequently it only makes sense to consider the problem in terms of a reduced time $\tau=k^{2} D t$, which takes the shift to smaller wave vectors at longer times into account. In terms of the reduced time the asymptotic decay reported in Ref. 7 has the form

$$
F(k, \tau) \sim \frac{k^{3}}{\tau^{3 / 2}}
$$

and so the long-time tail rapidly becomes (proportionately) smaller as $k$ decreases. The system studied in Ref. 7 is, however, atypical. Random media found in nature, or studied experimentally, are typically much more dense.

In this article we describe calculations of $F(k, t)$ for a particle diffusing in a more realistic system, namely, a model of a glass composed of impenetrable colloidal spheres. These spheres are, we imagine, surrounded by solvent but are themselves much larger than the solvent particles. The dynamics of the colloidal particles composing the glass is so slow that we can consider the structure to be fixed. In contrast, the diffusing particle, which we consider to be very much smaller than the colloidal spheres, diffuses through the solvent in the voids between the spheres. It could, for instance, be a smaller colloidal particle or a tracer molecule in the solvent. The VCF for the mobile particles will decay on time scales that are very short compared to the time it takes the particles to diffuse a distance comparable with the size of the particles composing the glass. The integral of the VCF over this short time scale gives the so-called "short-time" diffusion coefficient. At longer times, when particles have moved 
over distances comparable with the size of the colloidal particles composing the glass, diffusion is hindered by the presence of the glassy matrix and the particle will diffuse with a diffusion coefficient which is smaller than the short-time diffusion coefficient. This is the true, asymptotic, "long-time", diffusion coefficient. In our simulations we focus on the long-time diffusive motion of the mobile particle. We will not attempt to resolve the very short time scale, rather, we will input a value for the short-time diffusion coefficient, $D_{0}$, then see how this value is subsequently modified to its long time value as the particle moves through the glass. For very small diffusing particles, hydrodynamic interactions between the mobile particles and the particles composing the glass are negligible. Thus the short-time diffusion coefficient is equal to the diffusion coefficient in the pure solvent, i.e., the value it would take were the colloidal glass not present. In principle it is possible to ease this restriction, that the mobile particles are small compared to the characteristic size of the pores in the glassy matrix. To do so we would have to calculate the modification to the short-time diffusion coefficient due to hydrodynamic interactions, and we should take into account the reduced volume accessible to the diffusing particles because of their finite size. However, to a first approximation, a model in which the mobile particle is described as a point particle is adequate to describe the diffusion of tracer colloids in packed bed of larger colloids. In recent experiments ${ }^{9}$ the size ratio between the stationary and mobile colloids could be made as large as 71 .

The question that we address is whether the algebraic decay of the SDSF should be experimentally observable in such systems.

\section{DESCRIPTION OF THE MODEL}

We consider a point particle performing a random walk on a three dimensional lattice. Certain sites on the lattice are excluded by the presence of impenetrable colloidal spheres. The VCF for a particle performing a random walk, in the absence of the spheres, decays to zero in one time step. To enforce the condition that the short-time part of the VCF decays rapidly to zero we therefore need one step of the random walk to correspond to a distance much less than the radius of the colloidal spheres. The particular lattice we used was the face-centered hypercubic lattice projected onto three dimensions. A cubic lattice would formally suffice, but the face-centered hypercube has better symmetry properties and non-Gaussian effects intrinsic to the lattice are much smaller. For a given configuration of colloidal spheres we determine whether each site on the lattice is inside or outside a sphere. From this we construct a list of lattice links which connect sites outside spheres to sites inside spheres. These we refer to as boundary links. Any random walker which is traveling along such a link has its velocity reversed at half a time step. This ensures that the correct boundary conditions are imposed on the diffusing particle at the surface of the (impenetrable) spheres, i.e., the flux and the equilibrium density gradient are zero. The configurations of spheres we used consisted of 1000 nonoverlapping spheres at a volume fraction of $\phi=0.59$. This is typical for a colloidal glass. The configu- rations were generated by the slow compression of an initially random distribution, using standard Monte Carlo techniques.

For a given configuration of the colloidal glass, the best possible statistics we could obtain would come from averaging over all possible random walks in the system. To do this explicitly would be prohibitively time consuming but fortunately, by taking a given wave vector, the moment propagation method ${ }^{10}$ allows us to do this in one simulation. A detailed description of the application of the method to the calculation of the SDSF is described in Ref. 7 but, in summary, we proceed as follows. The lattice is initialized with a quantity we denote by $P_{t r}(\mathbf{r}, t)$,

$$
P_{t r}(\mathbf{r}, 0)=\frac{1}{n} \sum_{\mathbf{i}_{n b}} \exp (-i \mathbf{k} \cdot \mathbf{r})+\frac{1}{n} \sum_{\mathbf{i}_{b}} \exp (-i \mathbf{k} \cdot \mathbf{r}) .
$$

This quantity is then propagated in time according to

$$
P_{t r}(\mathbf{r}, t+1)=\frac{1}{n} \sum_{\mathbf{i}_{n b}} P_{t r}(\mathbf{r}+\mathbf{i}, t)+\frac{1}{n} \sum_{\mathbf{i}_{b}} P_{t r}(\mathbf{r}, t),
$$

where the summation is over all the $n$ lattice vectors $\mathbf{i}$. The subscript $b$ refers to vectors that are boundary links and the subscript $n b$ to those which are not. At any given time the SDSF, averaged over all possible random walks, is given by

$$
F(\mathbf{k}, t)=\frac{1}{N} \sum_{\mathbf{r}} P_{t r}(\mathbf{r}, t) \exp (i \mathbf{k} \cdot \mathbf{r}) .
$$

The summation over $\mathbf{r}$ consists of the $N$ sites on the lattice which lie outside the spheres.

\section{RESULTS}

We consider the results of simulations performed on a lattice consisting of up to $L^{3}=144^{3}$ lattice sites. Applying periodic boundary conditions we used the scheme described above to calculate $F(k, t)$. We only performed the simulations for times less than the minimum time it takes a particle to cross the simulation box. Thus there are no finite-size effects to consider. The units for the simulation were chosen such that the time step and lattice spacing were both unity, giving a (short time) diffusion coefficient, $D_{0}$, equal to $1 / 4$. The wave vector was chosen to lie along one of the Cartesian axes, and will be treated as a scalar, $k$. The product of the wave vector $k$ and the particle radius $R$ was used to define a dimensionless wave vector, $k^{*} \equiv k R / 2 \pi$. In these units wave vectors which probe length scales of the order of a colloidal particle radius correspond to $k^{*} \sim 1$. Non-Gaussian effects intrinsic to the lattice (which occur even in the absence of the colloidal spheres and take the form of the SDSF decaying exponentially but with a modified exponent), are limited to wave vectors greater than $2 \pi$ divided by the lattice spacing, i.e., $k^{*}>R .^{7}$ We therefore restrict our analysis to reduced wave vectors much less than $R$. There are errors associated both with representing the sphere on a lattice, and with representing the decay time for the short time component of the VCF by one step of a random walk. To ensure that these errors do not effect our conclusions we repeated the simulations using an increasing number of lattice sites to represent 


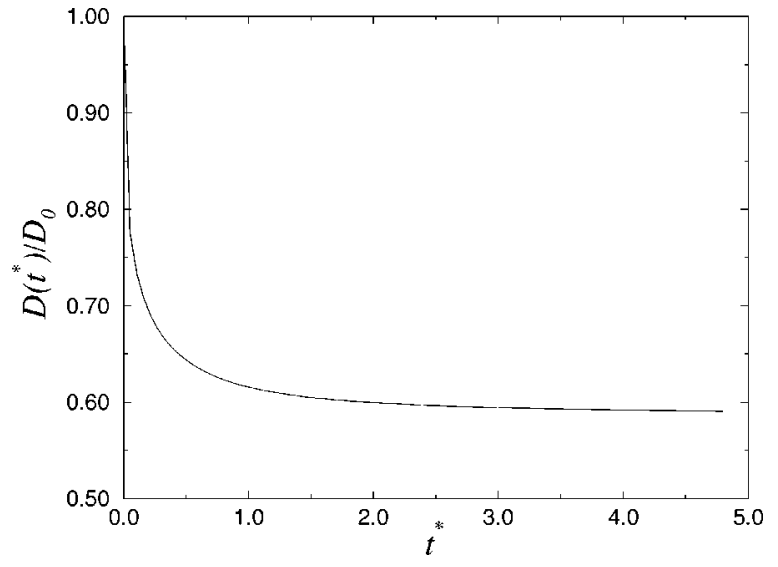

FIG. 1. The time dependent diffusion coefficient $D(t)$ divided by its short time value, $D_{0}$, and plotted as a function of a reduced time $t^{*}$ $\left(=t 6 D_{0} / R^{2}\right)$. A value of $t^{*}$ equal to unity corresponds to the time it typically takes the diffusing particle to displace a distance equal to the radius of the particles composing the glass.

a particle radius (keeping the volume fraction constant). This also has the effect of (proportionately) reducing the decay time for the short-time component of the VCF. Quantitatively, the difference between the values we obtained by representing a sphere radius with 7.5 lattice sites and representing a sphere radius with 9.5 lattice sites, for both the SDSF and the value of the long-time diffusion coefficient, were of the same magnitude as the errors associated with averaging over a number of configurations (a few percent). We therefore concluded that a sphere radius of 7.5 was adequate. All the results we show were obtained for this system. Three independent configurations of spheres were used and, for each configuration, the calculation was repeated with the wave vector oriented parallel to each of the three Cartesian axes, effectively giving a total of nine runs.

We start our analysis by looking at small $(\tau \ll 1)$ values of the reduced time $\tau$. Here we are looking at the very early stages of the decay of the SDSF where we expect Eq. (10) to be valid and any deviations from Fick's law to be small. We computed $D(t)$ and $B(t)$ by fitting our results for $F(k, t)$ to an equation with the form of Eq. (10). The results we obtained for $D(t)$ [which is directly related to the mean squared displacement by Eq. (11)] are plotted in Fig. 1 as a function of a reduced time $t^{*}$, defined as $t 6 D_{0} / R^{2}$. In these units a value of $t^{*}$ equal to unity represents the time it takes the diffusing particle to move a distance equal to the radius of the particles composing the glass. The time dependent diffusion coefficient, $D\left(t^{*}\right)$, reached an asymptotic value of $D / D_{0}=0.59 \pm 0.01$ after a time $t^{*} \sim 4$, i.e., once the particle has diffused a distance equivalent to a few radii of the glass particles. This value for $D / D_{0}$ is in line with values measured experimentally for packed beds of spherical particles at roughly the same density ${ }^{11}$. We stress that in order to calculate $D(t)$ it was essential to restrict ourselves to $\tau \ll 1$, i.e., to the initial decay of the SDSF. We performed an independent check that the $D(t)$ curve was correct by also calculating the VCF for the diffusing particles. From the VCF we can calculate the mean squared displacement directly, and hence the time dependent diffusion coefficient [from Eq. (5)]. The two

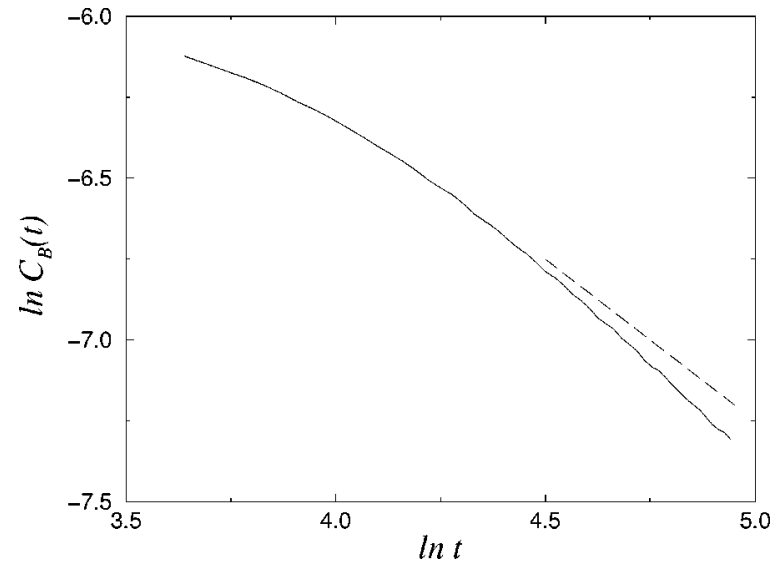

FIG. 2. The correlation function for the super-Burnett coefficient $C_{B}(t)$ (solid line). The dashed line has a slope -1 and is provided for comparison.

approaches [extracting $D(t)$ from the initial decay of the SDSF and from the time integral of the $\mathrm{VCF}]$ gave identical results, as they should. From the time dependent superBurnett coefficient, $B(t)$, we can obtain $C_{B}(t)$ $=(d / d t) B(t)$, the correlation function associated with the super-Burnett coefficient. In Fig. 2 we have plotted $C_{B}(t)$, which clearly decays asymptotically faster than $1 / t$. The time dependent super-Burnett coefficient must therefore be converging as predicted in Ref. 6 . The simulation results were not, however, sufficiently accurate to allow us to establish the long-time behavior of $C_{B}(t)$.

We now focus on the results for the self-dynamic structure factor at larger $(\tau>1)$ values of the reduced time. In Fig. 3 the self-dynamic structure factor is plotted in a linear$\log$ form. Clearly the SDSF is not decaying exponentially at longer times, otherwise the data in Fig. 3 would fall onto straight lines. Note that the apparent slowing down of the decay of the SDSF has nothing to do with the diffusion coefficient of the particle decreasing with time. As a specific example consider the wave vector $k^{*}=0.885$, the lowest curve shown in Fig. 3. For this wave vector the time $\tau=5$ is equivalent to a reduced time $t^{*}=0.97$ in Fig. 1 and the value

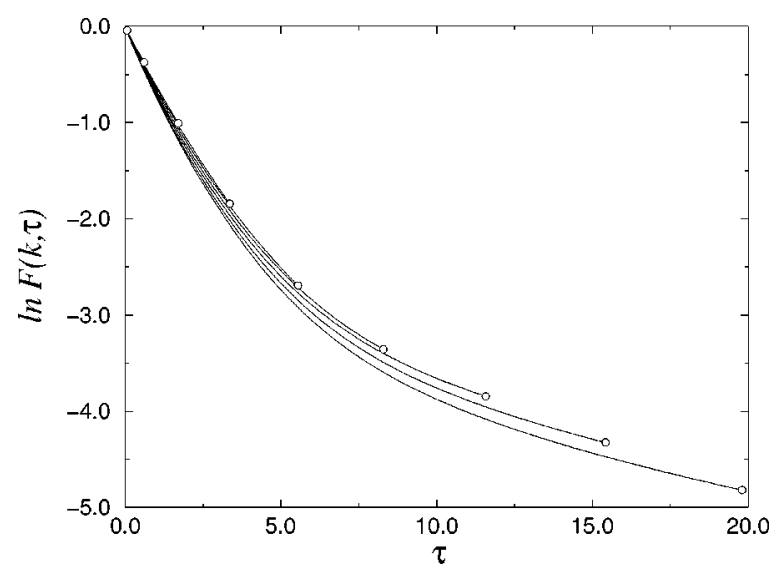

FIG. 3. Linear-log plot of $F(k, \tau)$ for different values of $k^{*}, k^{*}=0.052$, $0.156,0.260,0.365,0.496,0.573,0.677,0.781$, and 0.885 . The open circles denote the end of the simulation for increasing values of $k^{*}$ (from left to right). The errors are of the order of the circle size. 


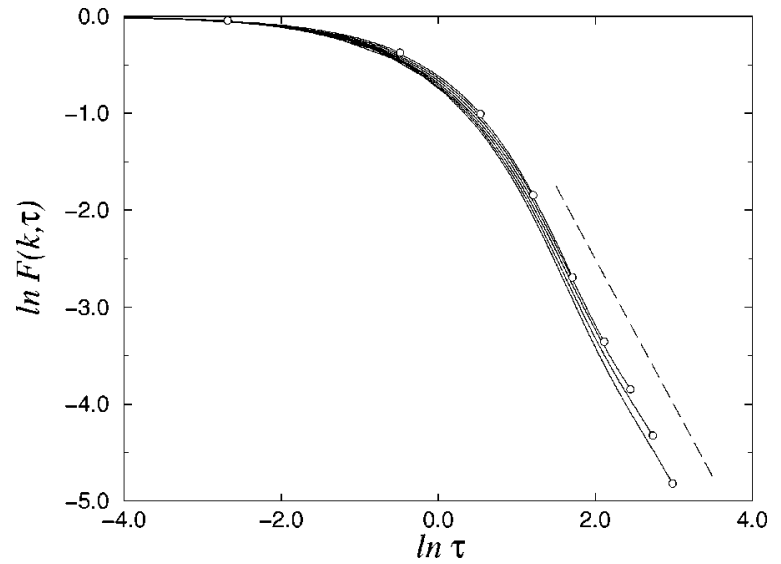

FIG. 4. Log-log plot of $F(k, \tau)$ for different values of $k^{*}, k^{*}=0.052,0.156$, $0.260,0.365,0.496,0.573,0.677,0.781$, and 0.885 . The open circles denote the end of the simulation for increasing values of $k^{*}$ (from left to right). The errors are of the order of the circle size. The dashed line has a slope -1.5 and is provided for comparison.

$\tau=15$ is equivalent to $t^{*}=2.91$. If we now examine Fig. 1 then clearly the change in $D(t)$ from $t^{*}=0.97$ to $t^{*}=2.91$ is rather small. In fact $D(t)$ decreases by just $2.5 \%$. On the other hand, the apparent decay of the SDSF [defined as the slope of the curves shown in Fig. 3, i.e., $d \ln F(k, t) / d t]$ decreases, over the identical time range, by a factor of $400 \%$. As we mentioned above, $D(t)$ has to be calculated from the initial rate of decay, i.e., for $\tau \ll 1$. The subsequent decay of the SDSF does not reflect a change in the value of $D(t)$, rather, the deviations from exponential behavior shown in Fig. 3 reflect deviations of $P(\mathbf{r}, t)$ from a Gaussian. It is somewhat surprising that such large deviations show up because we have restricted ourselves to times for which $F(k, \tau)$ is at least $1 \%$ of its initial value. If one were to look at the SDSF experimentally the decay could only be measured up until the time where its magnitude became of the same order as the statistical noise. If we assume a level of noise equal to about one-hundredth of the initial value of the SDSF then Fig. 3 shows roughly the range of values of $\tau$ that would be experimentally accessible. The simulations reported in Ref. 7 required much longer times before any significant deviation from exponential behavior was observed. Clearly, for this range of $k^{*}$, deviations from Fick's law should indeed be observable. The remaining questions are, could the true asymptotic decay be observed? And would the effect still be observable at even smaller values of $k^{*}$ ? The reason for addressing the latter point is that the only length scale in the system is the size of the particles. If we can establish the behavior of the SDSF for $k^{*} \ll 1$, i.e., over spatial length scales much longer than a particle radius, it would therefore be reasonable to assume that this represents the true small $k$ behavior. This is the most interesting case because it corresponds to particles undergoing large displacements in the colloidal glass. In Fig. 4 we have plotted the SDSF in log-log form. For comparison we also show the asymptotic decay of $F(k, \tau) \sim 1 / \tau^{3 / 2}$ that we expect based on the simulations reported in Ref. 7 and on the theory reported in Ref. 6. For sufficiently long times, the data are reasonably well represented by a power law decay. In fact, a linear fit to the

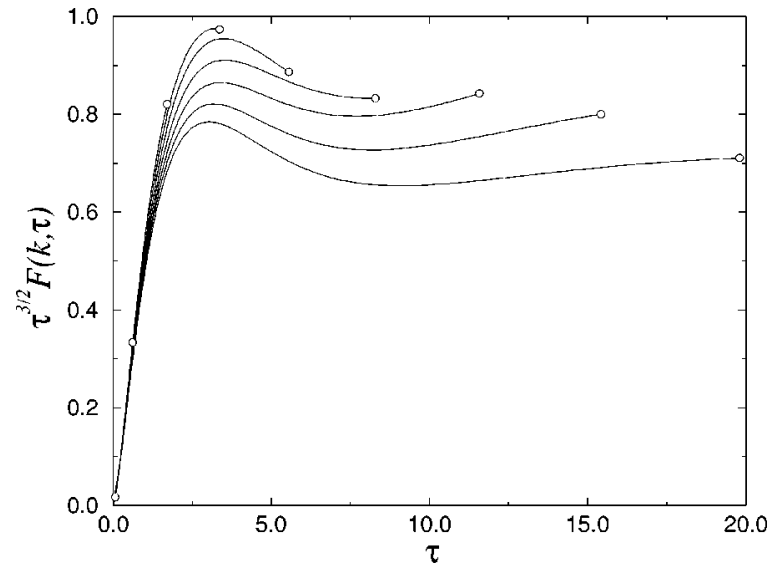

FIG. 5. Plot of $\tau^{3 / 2} F(k, \tau)$ for different values of $k^{*}, k^{*}=0.052,0.156$, $0.260,0.365,0.496,0.573,0.677,0.781$, and 0.885 . The open circles denote the end of the simulation for increasing values of $k^{*}$ (from left to right). The errors are of the order of the circle size.

long-time decay at $k^{*}=0.885$ yields a power law exponent of $-1.38 \pm 0.02$. A more accurate way of analyzing the simulation data is to plot $\tau^{3 / 2} F(k, \tau)$ and see if this function reaches a constant value. This we show in Fig. 5. The figure strongly suggests that the function is reaching a plateau value, indicating that asymptotically $1 / \tau^{3 / 2}$ decay is approached. However, the asymptote is not quite reached within the simulation time even at the largest wave vector we have plotted $\left(k^{*}=0.885\right)$. This is why the linear fit to the log-log plot yielded a value for the algebraic exponent slightly greater than the expected value of -1.5 . We now turn to our second question; does the effect decrease with decreasing wave vector? Figure 5 suggests not. The value of the coefficient characterizing the magnitude of the long-time tail (the plateau value in Fig. 5) does not appear to be decreasing. On the contrary, it appears if anything to increase slightly (although the data does not extend to long enough times for us to establish the precise form of the scaling). This is in contrast to the behavior at very low densities ${ }^{7}$ where the coefficient is proportional to $k^{3}$ and scales rapidly away with decreasing $k$. There is no reason to expect that the results obtained for these dense packings should be the same as the low density results. The analysis which predicts the scaling of the tail coefficient with $k$ is based on a single particle analysis. There will also be contributions from many particle interactions which this analysis neglects and which one would expect to become more important at higher volume fractions. Indeed, this is true of the VCF which displays a preasymptotic decay which strongly depends on the density and a asymptotic decay with an amplitude which also depends strongly on the density. ${ }^{5}$ Perhaps the most striking observation from Fig. 5 is just how little the dimensionless SDSF changes as a function of wave vector. This would suggest that, even for wave vectors smaller than those we have studied, the long-time tail in $F(k, \tau)$ should still make an experimentally observable contribution to the SDSF for a tracer particle in a colloidal glass. 


\section{CONCLUSIONS}

We studied the diffusion of a small particle in a colloidal glass and found numerical evidence that the super-Burnett coefficient converges, in line with theoretical expectations. Since the super-Burnett coefficient should characterize the lowest order correction to Fick's law this might suggest that any deviations from an exponential decay of the SDSF should be small and not relevant to the analysis of experimental results. However, we found that this was not the case because the long-time tail in SDSF is a large effect in densely packed systems and its influence should be clearly observable in an experiment. In contrast to earlier work, we also find that the magnitude of the tail does not decrease (proportionately) as the wave vector decreases. This suggests that the effect should be observable, not just for the relatively large values of the wave vector we have studied, but also for the much smaller values accessible to experiment but not to simulation.

\section{ACKNOWLEDGMENTS}

The work of the FOM Institute is part of the scientific program of FOM and is supported by the Nederlandse Or- ganisatie voor Wetenschappelijk Onderzoek (NWO). The research of Dr. Lowe has been made possible by a fellowship of the Royal Netherlands Academy of Arts and Sciences. Computer time on the CRAY-C98/4256 at SARA was made available by the Stichting Nationale Computer Faciliteiten (Foundation for National Computing Facilities). We would like to thank Henk van Beijeren and Jon Machta for useful comments, Ignacio Pagonabarraga, Richard Sear and Alfons van Blaaderen for critically reading the manuscript and Peter Bolhuis for providing the hard-sphere configuration.

${ }^{1}$ B. J. Alder and T. E. Wainwright, Phys. Rev. A 1, 18 (1970).

${ }^{2}$ M. H. Ernst, E. H. Hauge, and J. M. J. van Leeuwen, Phys. Rev. Lett. 25, 1254 (1970).

${ }^{3}$ J. R. Dorfman and E. G. D. Cohen, Phys. Rev. Lett. 25, 1257 (1970).

${ }^{4}$ M. J. Ernst and A. Weijland, Phys. Lett. 34A, 39 (1971).

${ }^{5}$ C. P. Lowe and A. J. Masters, Physica A 195, 149 (1993).

${ }^{6}$ M. H. Ernst, J. Machta, J. R. Dorfman, and H. van Beijeren, J. Stat. Phys. 34, 413 (1984).

${ }^{7}$ C. P. Lowe, D. Frenkel, and M. A. van der Hoef, J. Stat. Phys. 87, 1229 (1997).

${ }^{8} \mathrm{H}$. van Beijeren (private communication).

${ }^{9}$ S. G. J. M. Kluijtmans and A. P. Philipse (in preparation).

${ }^{10}$ D. Frenkel and M. H. Ernst, Phys. Rev. Lett. 63, 2165 (1989).

${ }^{11}$ J. J. Fried and M. A. Combarnous, Adv. Hydr. Sci 7, 169 (1971). 